\title{
Prediction of kinase inhibitor response using activity profiling, in vitro screening, and elastic net regression
}

Trish P Tran ${ }^{1}$, Edison Ong ${ }^{2}$, Andrew P Hodges ${ }^{1}$, Giovanni Paternostro ${ }^{1,2}$ and Carlo Piermarocchi ${ }^{2,3^{*}}$

\begin{abstract}
Background: Many kinase inhibitors have been approved as cancer therapies. Recently, libraries of kinase inhibitors have been extensively profiled, thus providing a map of the strength of action of each compound on a large number of its targets. These profiled libraries define drug-kinase networks that can predict the effectiveness of untested drugs and elucidate the roles of specific kinases in different cellular systems. Predictions of drug effectiveness based on a comprehensive network model of cellular signalling are difficult, due to our partial knowledge of the complex biological processes downstream of the targeted kinases.
\end{abstract}

Results: We have developed the Kinase Inhibitors Elastic Net (KIEN) method, which integrates information contained in drug-kinase networks with in vitro screening. The method uses the in vitro cell response of single drugs and drug pair combinations as a training set to build linear and nonlinear regression models. Besides predicting the effectiveness of untested drugs, the KIEN method identifies sets of kinases that are statistically associated to drug sensitivity in a given cell line. We compared different versions of the method, which is based on a regression technique known as elastic net. Data from two-drug combinations led to predictive models, and we found that predictivity can be improved by applying logarithmic transformation to the data. The method was applied to the A549 lung cancer cell line, and we identified specific kinases known to have an important role in this type of cancer (TGFBR2, EGFR, PHKG1 and CDK4). A pathway enrichment analysis of the set of kinases identified by the method showed that axon guidance, activation of Rac, and semaphorin interactions pathways are associated to a selective response to therapeutic intervention in this cell line.

Conclusions: We have proposed an integrated experimental and computational methodology, called KIEN, that identifies the role of specific kinases in the drug response of a given cell line. The method will facilitate the design of new kinase inhibitors and the development of therapeutic interventions with combinations of many inhibitors.

Keywords: Drug response predictions, Kinase inhibitors, Elastic net regression, High throughput screening, Drug combination therapies

\section{Background}

The important role of kinases in cancer biology [1] has spurred a considerable effort towards the synthesis of libraries of fully profiled kinase inhibitors, providing a map of the strength of each compound on a large number of its potential targets [2-4]. In particular, a recently published dataset has profiled several hundred kinase

\footnotetext{
* Correspondence: carlo@pa.msu.edu

${ }^{2}$ Salgomed Inc., Del Mar, CA 92014, USA

${ }^{3}$ Department of Physics and Astronomy, Michigan State University, East Lansing, Ml 48824, USA

Full list of author information is available at the end of the article
}

inhibitors using a panel of more than 300 kinases [4]. These profiled libraries define a network of interactions between drugs and their kinase targets [5], and represent a valuable resource for the development of new therapies. In this paper, we introduce a novel computational method that incorporates profiled libraries and in vitro measurements to predict the response of cells to previously untested drugs. Besides making prediction about the cellular response to drugs, the method identifies critical kinase targets and pathways that are statistically associated to drug sensitivity in a given cell line.

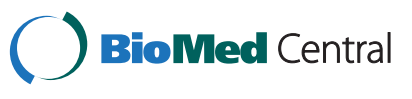


Statistical inference and regression methods in conjunction with gene expression or mutations have been used to identify specific biomarkers associated with an increased sensitivity/resistance to drugs. For instance, the sensitivity to PARP inhibitors of Ewing's sarcoma cells with mutations in the EWS gene and to MEK inhibitors in NRAS-mutant cell lines with AHR expression have been predicted using analysis of variance and the elastic net method [6] and then experimentally validated $[7,8]$. In these analyses, the statistical variable associated to drugs was represented by the half maximal inhibitory concentration $\left(\mathrm{IC}_{50}\right)$ in different cell lines. However, besides the $\mathrm{IC}_{50}$, there are many other types of information that characterize chemical compounds. These types of information can enhance the statistical analyses and improve the accuracy of predictions. For instance, a method to predict drugs sensitivity in cell lines based on the integration of genomic data with molecular physico-chemical descriptors of the drugs has been recently proposed [9]. Another useful type of information is the residual activity of kinases after interacting with a compound. Kinase profiling, patient genetic profiles, and sensitivity of primary leukemia patient samples to kinase inhibitors were recently used by Tyner et al. [10] to identify functionally important kinase targets and clarify kinase pathway dependence in cancer.

In this paper, the residual activity of kinases upon drug interaction is used to make predictions of the cellular response for in vitro experiments using an elastic net [6] regression approach. This regression method reduces the number of predictors to a minimum set, providing a clear picture of the kinases involved in the response of cell lines. A primary screen (single drug) and a secondary screen (two-drug combinations) are used as the training set for the regression. The two-drug screening exhibits a broader distribution in the response and provides a good level of predictability. In fact, the model based only on single drug response did not pass the statistical cross-validation test.

We are applying this Kinase Inhibitor Elastic Net (KIEN) method to predict cell viability of a lung cancer cell line (A549) and a normal fibroblast cell line (IMR-90) after drug treatment. We found that the regression can be improved through a logarithmic transformation on the data. Using the results of the regression, we identified a set of kinases that are strongly associated to a selective response of A549 and not IMR-90. Then, a pathway-based enrichment using Reactome [11] revealed ten significant pathways using this set of kinases, including axonal guidance and related semaphorin interactions as top hits.

This paper is organized as follows: Section In vitro screen of the kinase inhibitor library contains the experimental results of the primary and secondary in vitro screening corresponding to single drugs and two-drug combinations.
These experimental results and residual kinase activity are analyzed with Pearson's correlation in Section Analysis of correlations. This simple correlation analysis gives a first glance of the kinases that are statistically associated to a significant change in the viability of cancer and normal cell lines. In Section Elastic net regression, we introduce the elastic net approach and we present the results of a leaveone-out cross validation for predictions on single and pairs of drugs. We also present in this section the results obtained using the logarithmic transformation on the variables and a pathway enrichment analysis using Reactome [11]. The Discussion of the results is in Section Discussion, conclusions in Section Conclusions, and Materials and Methods in Section Materials and methods.

\section{Results}

\section{In vitro screen of the kinase inhibitor library}

Our methodology begins with the high-throughput screening of single drug and drug pair experiments. The 244 kinase inhibitors (KIs) of the EMD drug library were screened at $1000 \mathrm{nM}$ individually and the treatment lasted for 72 hours. To quantify a selective response of a cancer cell line with respect to a control normal cell line, we define the selectivity $S$ of a single drug or drug combination as

$$
S=\frac{v_{N}}{v_{C}}
$$

where $v_{N}$ indicates the viability of normal cells (IMR90) after treatment, and $v_{C}$ the viability of cancer cells (A549) after treatment. From the screening of the 244 KIs, the top hit was PDK1/Akt1/Flt3 Dual Pathway Inhibitor (CAS \# 331253-86-2) as ranked by selectivity (Figure 1). For the secondary screen, we used the PDK1/ Akt1/Flt3 Dual Pathway Inhibitor as the starting point and combined this compound with the other KIs as a drug pair combination. The dose of PDK1/Akt1/Flt3 Dual Pathway Inhibitor was studied to ensure proper dosing range and minimize toxicity. We used $125 \mathrm{nM}$, which maintains the normal cell line IMR-90's viability $>90 \%$ (Figure 2). For the other $243 \mathrm{KIs}$ we used the standard dose of $1000 \mathrm{nM}$. Several pairs in the secondary screen showed very high selectivity. The top hit from the secondary screen of the library was Alsterpaullone 2-cyanoethyl (CAS \# 852529-97-0) with a selectivity of $\mathrm{S}=6.14$ for the pair (Figure 3).

\section{Analysis of correlations}

In our second step, we analyzed the Pearson's correlation of the primary and secondary screening with a published dataset [4] containing target profiles for 140 kinase inhibitors. Therefore, even though we had a library of 244 KIs in the experimental screening, we were limited to utilizing $140 \mathrm{KIs}$ for the analysis. For each inhibitor, 


\section{Top Ten Hits of Primary Screen}

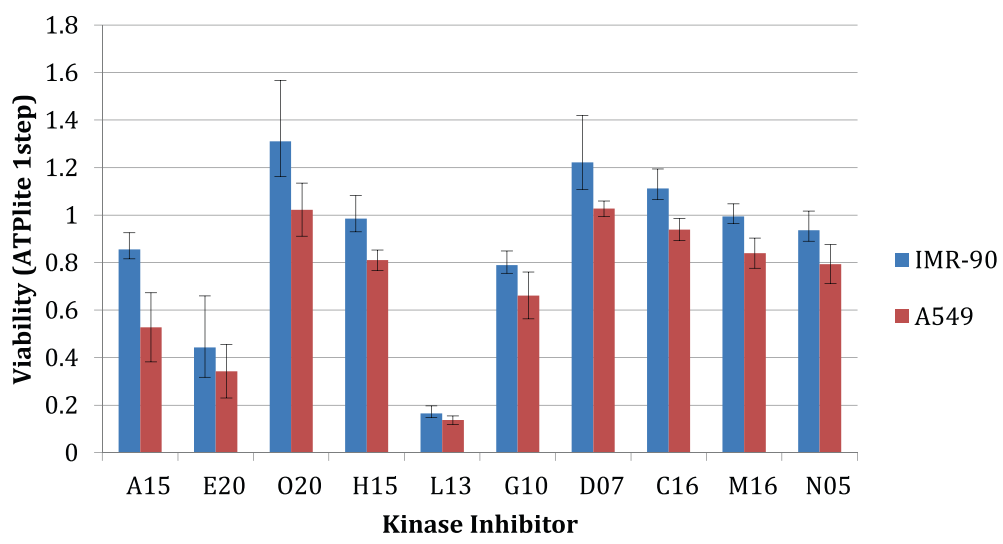

Figure 1 Primary screen results of the top ten most selective kinase inhibitors. Drugs are ranked based on the IMR-90 to A549 viability ratio. The 3 digit codes identify the compounds: A15: PDK1/Akt1/Flt3 Dual Pathway Inhibitor (CAS 331253-86-2); E20: Cdk/Crk Inhibitor (CAS 784211-09-2); O20: SU9516 (CAS 666837-93-0); H15: MEK1/2 Inhibitor II (CAS 212631-61-3); L13: PI 3-Ka Inhibitor VIII (CAS 372196-77-5); G10: Fascaplysin, Synthetic (CAS 114719-57-2); D07: Cdk2 Inhibitor II (CAS 222035-13-4); C16: Cdk1/2 Inhibitor III (CAS 443798-55-8); M16: GSK3b Inhibitor XII, TWS1 19 (CAS 601514-19-6); N05: Reversine (CAS 656820-32-5). The chemical structure of these compounds is given in a Additional file 2.

the dataset provides the residual activity $(0 \leq A \leq 1)$ of 291 kinases after drug treatment. This quantity is a measure of the strength of inhibition of a drug on each kinase.

For each kinase $k$, we calculate the Pearson's correlation, $C_{k}$, between the selectivity $S_{i}$ and the activities $A_{k, i}$, with $i \in\{1, \ldots, M\}$ indicating the single drug or drug pair in the set. For drug pairs, the activity is estimated as a product of the residual activities of the two drugs. The kinases are then ranked based on the $p$-value of their correlation with selectivity, and we calculate the False Discovery Rate (FDR) adjusted $p$ value [12]. The list of kinases mostly correlated to the selectivity from the primary and secondary screen are listed in Table 1 . We also did calculations of the correlation between the normal or cancer cell viability and the activities. The results for the top kinase-viability correlations for the primary and secondary screen are shown in the supplementary materials (Additional file 1: Table S1).

\section{Elastic net regression}

Next, we build a regression model that predicts the response of a cell line to a drug or drug combination $i$. The response we predict is the normal and cancer cell viability, from which the selectivity can be derived. For this purpose, we define a regression problem in which we use the residual activity of the kinase $k$ under the effect of drug $i$, which we indicate as $A_{k, i}$, as predictors of the viability. The response can be written as

$$
v_{i}=\beta_{0}+\beta_{1} A_{1, i}+\ldots+\beta_{p} A_{p, i}
$$

A fitting procedure based on a training set of measurements produces the coefficients $\left(\beta_{0}, \beta_{1}, \ldots, \beta_{p}\right)$. Equation

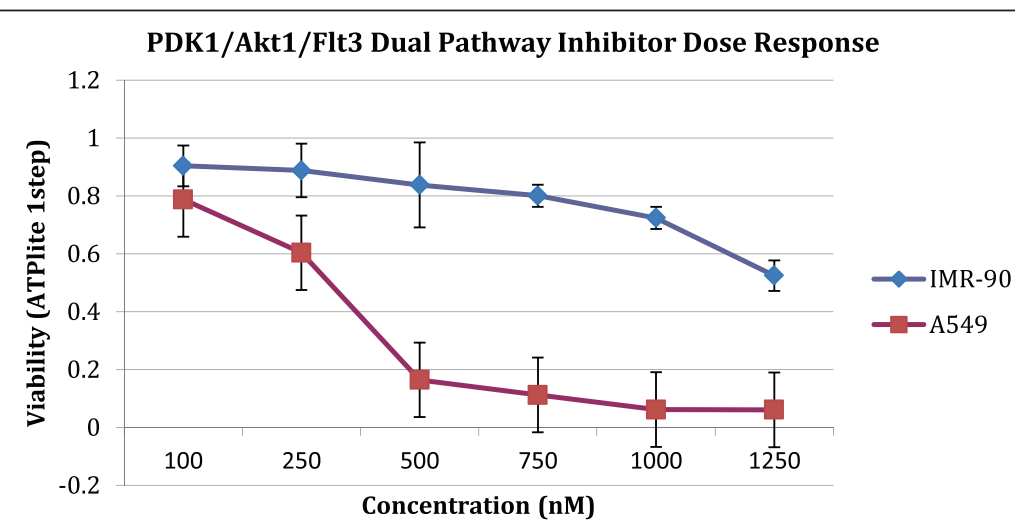

Figure 2 Dose response curve of PDK1/Akt1/FIt3 dual pathway inhibitor. Different doses of PDK1/Akt1/Flt3 Dual Pathway Inhibitor were tested to measure the response of A549 to the drug. For the secondary screen we selected 125nM to ensure low toxicity on the normal cell line. 


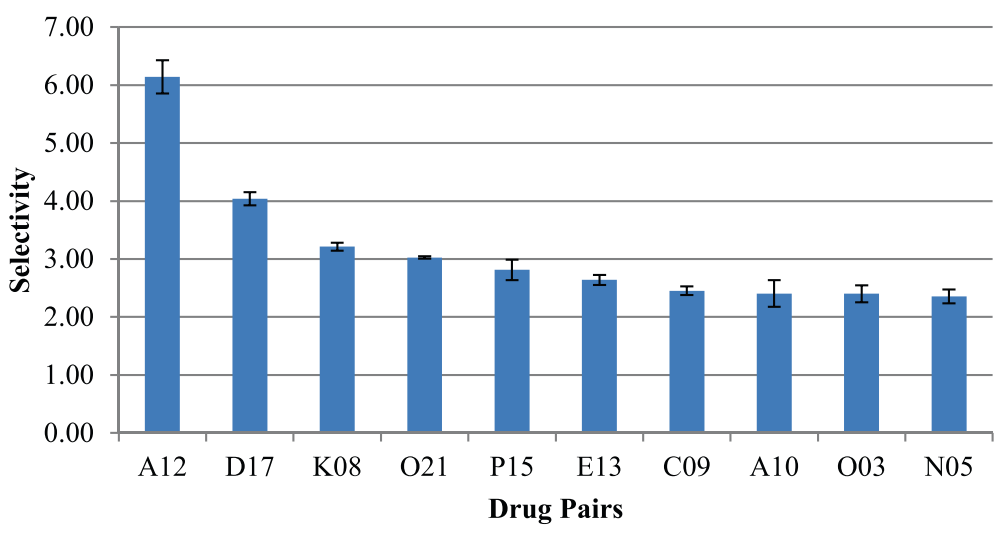

Figure 3 Secondary screen results of the top ten most selective drugs (1000 nM) when paired with PDK1/Akt1/Flt3 dual pathway inhibitor at $125 \mathrm{nM}$. Selectivity is the IMR-90 to A549 viability ratio, as defined in Section 2.1. The 3 digit codes identify the compounds: A12: Alsterpaullone, 2-Cyanoethyl (CAS 852529-97-0); D17: Cdk2/9 Inhibitor (CAS 507487-89-0); K08: K-252a, Nocardiopsis sp. (CAS 97161-97-2); O21: Staurosporine, Streptomyces sp. (CAS 62996-74-1); P15: WHI-P180, Hydrochloride (CAS 211555-08-7); E13: Gö 6976 (CAS 136194-77-9); C09: Compound 56 (CAS 171745-13-4); A10: Alsterpaullone (CAS 237430-03-4); O03: AG 1478, Selective inhibitor of epidermal growth factor receptor (EGFR) protein (CAS 175178-82-2); N05: Reversine (CAS 656820-32-5). The chemical structure of these compounds is given in a Additional file 2.

(1) can then be used to predict the viability of a new drug that has not been tested, but of which the profiling information is available. Note that we are integrating two different types of data: kinase profiling data is obtained through enzymatic assays that probe directly the interaction between drug and kinases, while the in vitro cell response data is the result of complex signaling that involves many pathways downstream of the affected kinases. The coefficients $\beta_{k}$ can be seen as a measure of the sensitivity of a given cell line due to alterations in the activity of kinase $k$.

It is well known that the least square method does not perform well in the case of linear regression with many predictors. In our case, we would like to use a database of drugs that have been profiled on about 300 kinases.
However, it would be desirable to select and keep in the final model a minimal set of the kinases that provide a simple model, useful to gain biological insight. The lasso technique [13] is a powerful method to reduce the number of predictors by imposing a penalty on the regression coefficients. However, in the presence of a group of kinase predictors with strong mutual correlation, the lasso could select only one kinase predictor from the group while missing the others. To prevent this problem, our method uses the elastic net approach. This method incorporates the lasso penalty as well as a ridge penalty to keep the regression coefficients small without completely removing them [6]. The weights of the ridge and lasso penalties in the least square procedure can be optimized for best performance of the method.

Table 1 Correlations between selectivity and kinase activity from primary and secondary screening

\begin{tabular}{|c|c|c|c|c|c|}
\hline \multirow{2}{*}{$\begin{array}{l}\text { Kinase } \\
\text { Primary screening }\end{array}$} & \multirow[t]{2}{*}{ Selectivity corr } & \multirow[t]{2}{*}{ FDR } & Kinase & Selectivity corr & \multirow[t]{2}{*}{ FDR } \\
\hline & & & \multicolumn{2}{|c|}{ Secondary screening } & \\
\hline$\overline{P R K C Z}$ & 0.451 & $2.28 \mathrm{E}-08$ & TGFBR2 & -0.501 & $8.29 \mathrm{E}-08$ \\
\hline DMPK & 0.435 & 7.75E-08 & CDK4 & -0.412 & $6.40 \mathrm{E}-05$ \\
\hline STK39 & 0.430 & $1.15 \mathrm{E}-07$ & CDC42BPB & -0.409 & $6.40 \mathrm{E}-05$ \\
\hline EPHA8 & 0.420 & $2.33 \mathrm{E}-07$ & RIPK2 & -0.399 & 7.73E-05 \\
\hline ADRBK2 & 0.399 & $1.01 \mathrm{E}-06$ & DSTYK & -0.369 & 0.000413 \\
\hline PRKACG & 0.396 & $1.27 \mathrm{E}-06$ & ACVRL1 & -0.368 & 0.000413 \\
\hline CAMK4 & 0.394 & $1.45 \mathrm{E}-06$ & PAK1 & -0.367 & 0.000413 \\
\hline MAP2K2 & 0.393 & $1.53 \mathrm{E}-06$ & MAPKAPK2 & -0.364 & 0.000413 \\
\hline ADRBK1 & 0.392 & $1.62 \mathrm{E}-06$ & PAK7 & -0.359 & 0.000424 \\
\hline PNCK & 0.382 & $3.29 \mathrm{E}-06$ & CDK1 & -0.357 & 0.000429 \\
\hline
\end{tabular}

A negative correlation indicates that inhibition of that particular kinases is associated to a higher selectivity. The top two hits with negative correlation, TGFBR2 and CDK4 are known to have an important role in cell proliferation, invasion and metastasis in lung adenocarcinoma [21,22]. 
We show in Figure 4(a) and (b) the results of a leave one out cross validation (LOOCV) method for the primary (a) and secondary screen (b). For each of the 140 drugs, we apply the elastic net method using the remaining 139 drugs and then we compare the result to the measured value. This cross validation method is a particular case of the more general $k$-fold cross validation procedure in which $k$ is equal to the size of the training set [14]. The cross LOOCV shows that the information contained in the primary screen is not sufficient to define a predictive model. The fact that some kinases in Table 1 show some significant correlation with the response when considered individually is in general not a sufficient condition for defining a predictive, multiple regression model. On the other hand, the secondary screen is able to reproduce the viability of many drugs, especially the ones with the stronger effect on both cell lines. Overall, the data from the secondary screen presents a much broader distribution with a tail representing a few drug combinations particularly effective. The regression works better in identifying these highly effective pairwise combinations and the relative ranking of their strengths. Data is not particularly informative for drugs and drug pair combinations that are not effective, which concentrate in the neighborhood of $\sim 1$.

Data transformations can represent a powerful strategy to improve regression. We applied a logarithmic transformation, which is consistent with the hypothesis of an independent action on the different kinases on the total viability. In this case we assume that the viability can be rewritten in the form

$$
v_{i}=e^{\beta_{0}}\left(A_{1, i}\right)^{\beta_{1}} \cdot\left(A_{2, i}\right)^{\beta_{2}} \cdot \ldots \cdot\left(A_{p, i}\right)^{\beta_{p}} .
$$

By applying a $\log$ transformation on both sides of Eq. (2) we reduce the problem to a linear regression, to which the elastic net strategy can be applied. We show in Figure 5 the results of the LOOCV for the primary and secondary screen using the logarithmic data transformation. As in the linear case, we find that the method fails the cross validation procedure if we use data from the primary screen, while the secondary screen with $\log$ transformed data gives better $R^{2}$.

In addition to a regression model that can be used to predict the efficacy of drugs that have not been tested, the $\beta_{i}$ coefficients can be used to rank kinases in terms of their relevance in the regression. Therefore, these coefficients identify the kinases whose inhibition is associated to a decrease in the cell viability. A ranking based on the differential $\beta_{i}^{C}-\beta_{i}^{N}$, where the index $N$ and $C$ identify the regression model of the cancer and normal cells, gives insight on specific pathways important for a selective response of cancer cells. Table 2 shows a list of kinases ranked in terms of $\left|\beta_{i}^{C}-\beta_{i}^{N}\right|$, where the coefficients have been obtained using the logarithmic data transformation on the secondary screen.

In order to test whether selected pathways were significantly enriched for the identified kinase genes in Table 2, a pathway-based enrichment analysis was
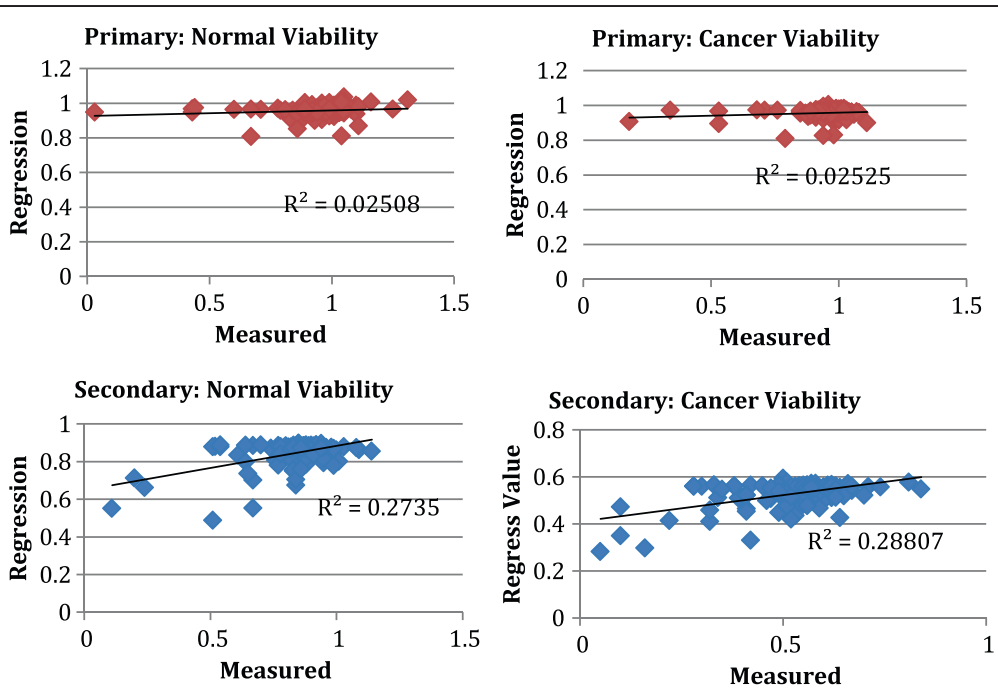

Figure 4 Leave-one-out Cross Validation of the elastic net regression model based on the primary (top) and secondary (bottom) screens for normal and cancer cell lines. Each of the 140 point in these figures corresponds to one of the 140 drug. "Regression" refers to the viability predicted by the regression model using all data from the other 139 drugs as training set, while "Measured" refers to the actual viability measured for the drug or drug combination. Note that only the secondary screen leads to predictive models with significant $R^{2}$ for the two cancer cell types. 

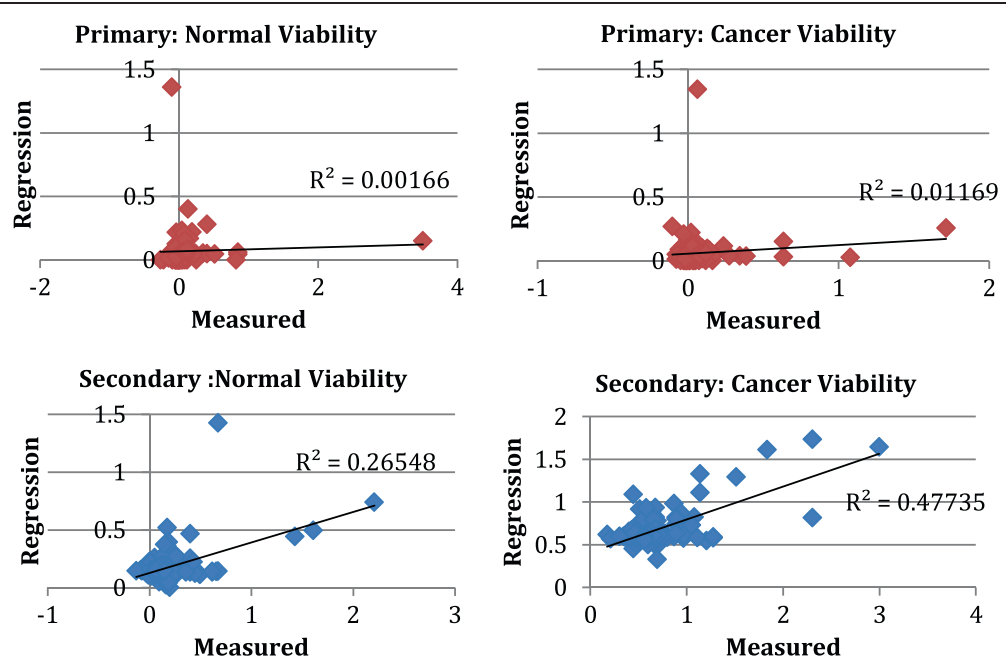

Figure 5 Leave-one-out Cross Validation of the elastic net regression model based on the primary (top) and secondary (bottom) screens for normal and cancer cell lines after logarithmic transformation on the data. Each of the 140 point in these figures corresponds to one of the 140 drugs. "Regression" refers to - log of the viability predicted by the regression model using all data from the other 139 drugs as training set, while "Measured" refers to -log of the actual viability measured for the drug or drug combination. Note that, as in Figure 4, only the secondary screen leads to predictive models with significant $R^{2}$ for both cell types. The $R^{2}$ for the Cancer cell lines is considerably better using the log transformation.

conducted using the results from the elastic net kinase analysis and Fisher exact tests. Ten pathways from Reactome were identified as significant $(p<0.05)$ using this kinase list, including axon guidance, activation of Rac, and semaphorin interactions as top hits (Table 3).

\section{Discussion}

Drug-kinase profiling represents a controller-target network [5] that when combined with in vitro testing, can be used in regression models to predict drug response and to identify pathways statistically associated to drug sensitivity. Network methods in biology are often based on the analysis of large datasets from high-throughput experiments. An example is given by gene regulatory networks, which presents many challenges either when restricted to a homogeneous set of data $[15,16]$ or when it includes different classes of data [17-20]. In our KIEN method, information from the drug-target network and experimental query of the biological system are integrated. The goal is not a reconstruction of a regulatory network, but to identify a set of kinases linked to a therapeutic response in a given cell line. In order to establish associations, the system has to be perturbed by the use of kinase inhibitor drugs. The response to these single drugs or drug combinations becomes a training set that when combined with the kinase profiling, can lead to predictions.

The elastic net method is one of the most widely used regularization techniques. Regularization techniques are used in statistical and machine learning models to achieve an optimal tradeoff between accuracy and simplicity. Simplicity makes a model less prone to overfitting and more likely to generalize. In our analysis, we found that the elastic net regressions based on single drug responses were not successful, while drug pair data provided statistically significant predictions. A possible explanation for this finding is the following: single drugs might be less able to overcome the robustness of biological networks [5]. The phenotypic signal is therefore blunted and not easily measured. If a second drug is added, any compensatory capacity is already stretched and the effects from the inhibition of each kinase can be seen more clearly. Using data from drug pairs, we found that noise can be better filtered out and stronger statistical associations between kinases and therapeutic response are revealed. Clearly, if a different training set with higher variance in efficacy measures were used in the primary screen, it is likely that also single drug in vitro response would have given a significant predictive model.

We identified several kinases that are implicated in lung cancer that gives biological significance to our KIEN method. In particular, TGFBR2 appears as a top hit both in the correlation and in the elastic net methods. This finding is consistent with recent siRNA experiments on A549 cell lines [21], which demonstrated that silencing of this receptor reduces cell proliferation, invasion, and metastasis. The Cyclin-dependent kinase 4 (CDK4) appears as a second top target in the correlation analysis, and is also highly significant in the KIEN analysis. Experiments using lentiviral-mediated shRNA to inhibit CDK4 in A549 have shown inhibited cell cycle 
Table 2 Kinases with the highest difference in the regression coefficients for the log transformed data of the secondary screen

\begin{tabular}{|c|c|c|c|}
\hline Kinase & $\begin{array}{l}\text { Cancer beta } \\
\text { coefficient }\end{array}$ & $\begin{array}{l}\text { Normal beta } \\
\text { coefficient }\end{array}$ & Difference \\
\hline TGFBR2 & 0.061 & 0.000 & 0.061 \\
\hline EGFR & 0.060 & 0.000 & 0.060 \\
\hline PHKG1 & 0.051 & 0.014 & 0.037 \\
\hline RIPK2 & 0.032 & -0.002 & 0.034 \\
\hline PRKG2 & 0.012 & 0.045 & 0.033 \\
\hline CDK4 & 0.021 & -0.008 & 0.029 \\
\hline MAP3K10 & 0.038 & 0.014 & 0.024 \\
\hline MARK4 & 0.000 & 0.022 & 0.022 \\
\hline PAK1 & 0.025 & 0.004 & 0.021 \\
\hline MAP4K5 & 0.021 & 0.000 & 0.021 \\
\hline MARK2 & 0.006 & 0.026 & 0.021 \\
\hline MARK3 & 0.000 & 0.020 & 0.020 \\
\hline TBK1 & 0.012 & 0.031 & 0.020 \\
\hline ERBB2 & 0.021 & 0.001 & 0.019 \\
\hline NUAK1 & -0.029 & -0.010 & 0.019 \\
\hline ULK2 & 0.018 & 0.000 & 0.018 \\
\hline MYLK2 & -0.024 & -0.006 & 0.018 \\
\hline MAP4K4 & 0.004 & -0.014 & 0.018 \\
\hline CDK5 & 0.002 & -0.016 & 0.018 \\
\hline GSK3B & 0.021 & 0.004 & 0.017 \\
\hline PAK2 & 0.019 & 0.002 & 0.017 \\
\hline CDC42BPB & 0.023 & 0.006 & 0.017 \\
\hline DSTYK & 0.006 & -0.010 & 0.016 \\
\hline RPS6KA2 & 0.000 & -0.016 & 0.016 \\
\hline FGFR1 & -0.004 & 0.012 & 0.016 \\
\hline PAK7 & 0.015 & 0.000 & 0.015 \\
\hline PIM1 & -0.015 & 0.000 & 0.015 \\
\hline CDK3 & 0.015 & 0.000 & 0.015 \\
\hline IRAK1 & -0.002 & -0.017 & 0.015 \\
\hline
\end{tabular}

A larger difference is associated with a selective response of A549 upon inhibition. Note that in addition to TGFB2R and CDK4, which were identified with the correlation approach of Table 1, additional kinases known to have an important role in lung cancer such as EGFR [24,25] and PHKG1 [26] are found using the elastic net approach.

progression, suppressed cell proliferation, colony formation, and migration [22], and there is an ongoing clinical trial using a CDK4/6 inhibitor in lung cancer [23]. The KIEN analysis identified EGFR, which is known to be overexpressed in the majority of nonsmall cell lung cancers [24]. Furthermore, RNAi experiments targeting EGFR demonstrated cancer growth suppression in A549 xenograft in mice [25]. The third kinase in Table 2, PHKG1 has also been found to be upregulated in human tumor samples, including lung
Table 3 Reactome pathways with significant representation of kinases from the regression analysis

\begin{tabular}{lllll}
\hline Path ID & Path name & $\mathbf{N}_{\mathbf{S}}$ & $\mathbf{N}_{\mathbf{T}}$ & $\mathbf{p}$-val \\
\hline 422475 & Axon guidance & 9 & 31 & 0.005 \\
428540 & Activation of Rac & 3 & 5 & 0.008 \\
373755 & Semaphorin interactions & 4 & 10 & 0.011 \\
376176 & Signaling by Robo receptor & 3 & 7 & 0.024 \\
1266738 & Developmental Biology & 8 & 39 & 0.026 \\
445144 & Signal transduction by L1 & 4 & 13 & 0.030 \\
373760 & L1CAM interactions & 4 & 14 & 0.040 \\
193639 & p75NTR signals via NF-kB & 2 & 4 & 0.051 \\
209543 & p75NTR recruits signaling complexes & 2 & 4 & 0.051 \\
389359 & CD28 dependent Vav1 pathway & 2 & 4 & 0.051 \\
\hline
\end{tabular}

$\mathrm{N}_{\mathrm{s}}$ indicates the number of kinases that are found significant in the regression analysis, while $N_{T}$ is the total number of kinases in the pathway. The top ten pathways with Fisher exact test $p<=0.051$ are shown. These pathways are identified from 518. Reactome pathways containing at least one of the kinases identified in Table 2. The 9 kinases in the axon-guidance pathway are EGFR, PAK1, ERBB2, CDK5, GSK3B, PAK2, RPS6KA2, FGFR1 and PAK7.

adenocarcinoma, and aberrations in its gene copy number is a feature of many human tumors [26].

The pathway-based enrichment provides a broader view on the role of the kinases identified by our method in Table 2. Among the top three pathways shown in Table 3 are activation of Rac and Semaphorin interactions. Rac proteins play a key role in cancer signaling and they belong to the RAS superfamily [27]. We also identified a set of semaphorins in our analysis that is represented in the top significantly enriched pathways. Semaphorins, previously known as collapsins, are a set of proteins containing a 500-amino acid sema domain among others (including PSI and immunoglobulin type domains), which can be transmembranous or secreted [28]. It is known that Sema3E cleavage promotes invasive growth and metastasis in vivo [28]. These genes also have selective targeting by Rac and Rho family members. This generates hypotheses of possible pathways that could be targeted therapeutically. However, these hypotheses need to be validated by further experiments with different inhibitors for the same targets or with alternative methods, e.g. using siRNA.

\section{Conclusions}

We have introduced an integrated experimental and computational methodology that identifies the role of specific kinases in the drug response of a given cell line. The key element of our KIEN methodology is a multiple regression procedure that uses in vitro screen data as a training set. If a new library of kinase inhibitor compounds were to be synthetized and profiled, then our model would be able to immediately estimate the effect of these drugs on in vitro experiments on a given cell line. We have shown an application to a lung cancer cell 
line, but our method can be extended to different cell lines. The method will facilitate the design of new kinase inhibitors and the development of therapeutic interventions with combinations of many inhibitors [29]. The procedure could be extended to three drug combinations, if measurements for these larger combinations were available. Finally, the method could be extended to regression models that are specific of cancer cells with the same set of mutations, or it could be directly used with patient-derived primary cells to identify a personalized treatment.

\section{Materials and methods Materials}

The primary screening of a kinase inhibitor (KI) library comprised of 244 KIs was purchased from EMD Chemicals, and diluted with DMSO to $2 \mathrm{mM}$ concentrations for high-throughput screening purposes. The KI library was stored at $-80^{\circ} \mathrm{C}$. Additionally, PDK1/Akt1/Flt3 Dual Pathway Inhibitor (CAS \# 331253-86-2) was ordered from EMD. Only 140 out of 244 were used in the drugtarget network reconstruction because the drug profiling information was available only for these compounds. One kinase inhibitor known to affect the kinase targets indirectly was excluded. We provide in Additional file 2 the chemical structure of kinase inhibitors with highest selectivity in the primary and secondary screening.

\section{Cell culture}

Cell lines IMR-90 (normal lung fibroblast) and A549 (lung adenocarcinoma) were cultured in RPMI 1640 (Hyclone) supplemented with 10\% Canadian characterized fetal bovine serum (Hyclone), 1\% $200 \mathrm{mM}$ L-glutamine (Omega), and $1 \%$ penicillin/streptomycin (Omega). The media for the cells were renewed every 3 days and kept at $80-90 \%$ confluency. Cells were maintained in a humidified environment at $37^{\circ} \mathrm{C}$ and $5 \% \mathrm{CO}_{2}$.

\section{Kinase inhibitor experiments}

IMR-90 (1500 cells/well) and A549 (750 cells/well) were seeded on 384-well microplates (Grenier Bio-One) and incubated for 3 hours before the addition of kinase inhibitor(s). The reason that IMR-90 was seeded at double the cell density of A549 is due to the difference in cell division. IMR-90's doubling time is $36-48$ hours whereas A549's is 22 hours. We wanted to make sure that the cells have divided at least once during the $72 \mathrm{hr}$ drug treatment. Furthermore, both A549's and IMR-90's final confluency at $72 \mathrm{hrs}$ is $90-95 \%$ and within the range of the ATPlite 1step assay. Additional file 1: Figures S1 and S2 show the growth curve for both cell lines. IMR-90 and A549 cell lines were tested on the same day with three replicates and the experiment was repeated three times with randomized well positions to reduce biases.
ECHO 555 Liquid Handler (Labcyte) was used to dispense nanoliter volumes of each KI to 384-well plates with cells attached (wet dispense). The final volume in the plate is $40 \mathrm{uL}$ and cells were incubated for 72 hours with KI treatment.

\section{ATP measurements}

ATPlite 1Step (Perkin Elmer) was used to evaluate the cell number and cytotoxicity. ATP measurements were done by dispensing $20 \mathrm{uL}$ of the ATPlite 1Step solution to each well to a final volume of $60 \mathrm{uL}$. The plate was placed on a shaker at $1100 \mathrm{rpm}$ and the luminescence activity was detected by Analyst GT Plate Reader. The percent (\%) of control is the quantity of ATPlite 1step measurement of the treated versus the untreated wells of each individual cell type. The ATP standard was prepared with culture media to final volume of $40 \mathrm{uL}$, and $20 \mathrm{uL}$ of ATPlite 1step reagent was added. Additional file 1: Figure S3 shows the ATP standard curve. The plate was read immediately.

\section{Computational methods}

Correlations between selectivity/viability and kinase activity were calculated using the python scipy linregress function, which also provide $p$-values. Ranking the $\mathrm{p}$-values and directly applying the Benjamini-Hochberg procedure gave us the FDR values. The elastic net regression was carried out using the Scikit-learn package [30] which finds the coefficients $\beta$ that minimize the function

$$
F=\frac{1}{2 M}\|\nu-A \beta\|_{2}^{2}+\alpha \rho\|\beta\|_{1}+\frac{1}{2} \alpha(1-\rho)\|\beta\|_{2}^{2}
$$

where $v$ is the vector of the observed viabilities and $A$ is the matrix containing the residual activity of the kinases from the profiling, and $M$ is the total number of drugs or drug combinations used. The parameters $\alpha$ and $\beta$ determine the relative weights of the lasso and ridge penalties quantified using $L^{1}\left(\|\left.\right|_{1}\right)$ and $L^{2}\left(\|\|_{2}\right)$ norm, respectively. We used $\alpha=0.15$ and $\rho=0.01$ in the results of Figures 4 and 5 and in Table 2. We also tried other values of these parameters, which did not give a significant difference in the results.

\section{Pathway-based enrichment}

Reactome pathways were downloaded using a newer build of the 'biomaRt' library (v2.12.0) in Bioconductor/R (v2.15.0). Gene symbols from the kinase list were converted to Entrez gene identifier numbers ('entrezgene') and mapped against the gene ids in each Reactome pathway. For each pathway, the set of significant genes enriched within any given pathway was computed using a Fisher exact test. The procedure computes the significance (p-value) of observing significant kinases, as deemed significant by our method, within the selected 
pathway. These pathways are identified from 518 Reactome pathways. Given that our gene set consists entirely of kinases and would be generalized towards kinase-specific effects, the set of all kinases $(\sim 300)$ were selected for background adjustment and more sensitive enrichment of the pathways. This procedure was repeated for each pathway to generate p-values and pathway rankings. False discovery rate [FDR] values were later generated to further restrict significance.

\section{Additional files}

\section{Additional file 1: Prediction of kinase inhibitor response using activity profiling, in-vitro screening, and elastic net regression. Additional file 2: Chemical structure of drugs with the highest selectivity in the primary and secondary screen.}

\section{Competing interests}

The authors declare that they have no competing interests.

\section{Authors' contributions}

GP and CP proposed the concept, EO and CP wrote the software and analysed the results, APH performed the Reactome analysis, $T$ performed all experiments, CP and GP. wrote the manuscript. All authors read and approved the final manuscript.

\section{Acknowledgements}

We thank Dr. Anthony (Tony) Polverino for many discussions. We would like to acknowledge the NSF grant (CCF0829891) and the DOD/CDMRP Lung Cancer Research Program (grant W81XWH-12-1-0233) for support. The data sets supporting the results of this article are included within the article. Correspondence and requests for materials should be addressed to giovanni@sanfordburnham.org or carlo@pa.msu.edu.

\section{Author details}

${ }^{1}$ Sanford-Burnham Medical Research Institute, La Jolla, CA 92037, USA ${ }^{2}$ Salgomed Inc., Del Mar, CA 92014, USA. ${ }^{3}$ Department of Physics and Astronomy, Michigan State University, East Lansing, Ml 48824, USA.

Received: 30 April 2014 Accepted: 18 June 2014

Published: 25 June 2014

\section{References}

1. Cohen P: Protein kinases - the major drug targets of the twenty-first century? Nat Rev Drug Discov 2002, 1:309-315.

2. Fabian MA, Biggs WH, Treiber DK, Atteridge CE, Azimioara MD, Benedetti MG, Carter TA, Ciceri P, Edeen PT, Floyd M, Ford JM, Galvin M, Gerlach JL, Grotzfeld RM, Herrgard S, Insko DE, Insko MA, Lai AG, Lelias J-M, Mehta SA, Milanov ZV, Velasco AM, Wodicka LM, Patel HK, Zarrinkar PP, Lockhart DJ: A small molecule-kinase interaction map for clinical kinase inhibitors. Nat Biotech 2005, 23:329-336.

3. Karaman MW, Herrgard S, Treiber DK, Gallant P, Atteridge CE, Campbell BT, Chan KW, Ciceri P, Davis MI, Edeen PT, Faraoni R, Floyd M, Hunt JP, Lockhart DJ, Milanov ZV, Morrison MJ, Pallares G, Patel HK, Pritchard S, Wodicka LM, Zarrinkar PP: A quantitative analysis of kinase inhibitor selectivity. Nat Biotech 2008, 26:127-132.

4. Anastassiadis T, Deacon SW, Devarajan K, Ma HC, Peterson JR: Comprehensive assay of kinase catalytic activity reveals features of kinase inhibitor selectivity. Nat Biotechnol 2011, 29:1039-U1117.

5. Feala JD, Cortes J, Duxbury PM, McCulloch AD, Piermarocchi C, Paternostro G: Statistical Properties and Robustness of Biological Controller-Target Networks. PLoS One 2012, 7:e29374.

6. Zou H, Hastie T: Regularization and variable selection via the elastic net. J R Stat Soc B 2005, 67:301-320.

7. Garnett MJ, Edelman EJ, Heidorn SJ, Greenman CD, Dastur A, Lau KW, Greninger P, Thompson IR, Luo X, Soares J: Systematic identification of genomic markers of drug sensitivity in cancer cells. Nature 2012, 483:570-575.

8. Barretina J, Caponigro G, Stransky N, Venkatesan K, Margolin AA, Kim S, Wilson CJ, Lehar J, Kryukov GV, Sonkin D: The Cancer Cell Line Encyclopedia enables predictive modelling of anticancer drug sensitivity. Nature 2012, 483:603-607.

9. Menden MP, lorio F, Garnett M, McDermott U, Benes CH, Ballester PJ, Saez-Rodriguez J: Machine Learning Prediction of Cancer Cell Sensitivity to Drugs Based on Genomic and Chemical Properties. PLoS One 2013, 8(4):e61318

10. Tyner JW, Yang WF, Bankhead A, Fan G, Fletcher LB, Bryant J, Glover JM, Chang BH, Spurgeon SE, Fleming WH: Kinase pathway dependence in primary human leukemias determined by rapid inhibitor screening. Cancer Res 2013, 73:285-296.

11. Matthews L, Gopinath G, Gillespie M, Caudy M, Croft D, de Bono B, Garapati $P$, Hemish J, Hermjakob H, Jassal B: Reactome knowledgebase of human biological pathways and processes. Nucleic Acids Res 2009, 37:D619-D622.

12. Benjamini $Y$, Hochberg $Y$ : Controlling the false discovery rate: a practical and powerful approach to multiple testing. J R Stat Soc B 1995, 57:289-300

13. Tibshirani R: Regression shrinkage and selection via the lasso. I R Stat Soc B 1996, 58:267-288.

14. Kohavi R: A study of cross-validation and bootstrap for accuracy estimation and model selection. In Proceedings of the 14-th International joint Conference on artificial intelligence. Edited by Mellish CS. Los Altos, CA: Morgan Kaufmann; 1995:1137-1145.

15. De Smet R, Marchal K: Advantages and limitations of current network inference methods. Nat Rev Microbiol 2010, 8:717-729.

16. Marbach D, Prill RJ, Schaffter T, Mattiussi C, Floreano D, Stolovitzky G: Revealing strengths and weaknesses of methods for gene network inference. Proc Natl Acad Sci 2010, 107:6286.

17. Marbach D, Costello JC, Kuffner R, Vega NM, Prill RJ, Camacho DM, Allison KR, Kellis M, Collins JJ, Stolovitzky G, Consortium D: Wisdom of crowds for robust gene network inference. Nat Methods 2012, 9:796.

18. Bar-Joseph Z, Gerber GK, Lee TI, Rinaldi NJ, Yoo JY, Robert F, Gordon DB, Fraenkel E, Jaakkola TS, Young RA: Computational discovery of gene modules and regulatory networks. Nat Biotechnol 2003, 21:1337-1342.

19. Lemmens K, De Bie T, Dhollander T, De Keersmaecker SC, Thijs IM, Schoofs G, De Weerdt A, De Moor B, Vanderleyden J, Collado-Vides J: DISTILLER: a data integration framework to reveal condition dependency of complex regulons in Escherichia coli. Genome Biol 2009, 10:R27.

20. Reiss D, Baliga N, Bonneau R: Integrated biclustering of heterogeneous genome-wide datasets for the inference of global regulatory networks. BMC bioinformatics 2006, 7:280.

21. Xu CC, Wu LM, Sun W, Zhang N, Chen WS, Fu XN: Effects of TGF-beta signaling blockade on human A549 lung adenocarcinoma cell lines. Mol Med Rep 2011, 4:1007-1015.

22. Wu AB, Wu B, Guo JS, Luo WR, Wu D, Yang HL, Zhen Y, Yu XL, Wang H, Zhou Y, Liu Z, Fang WY, Yang ZX: Elevated expression of CDK4 in lung cancer. J Transl Med 2011, 9:38.

23. US NIH Clinical Trials registry and results database. http://clinicaltrials.gov. <http://clinicaltrials.gov/show/NCT01291017> (2014).

24. Brabender J, Danenberg KD, Metzger R, Schneider PM, Park JM, Salonga D, Holscher AH, Danenberg PV: Epidermal growth factor receptor and HER2-neu mRNA expression in non-small cell lung cancer is correlated with survival. Clin Cancer Res 2001, 7:1850-1855.

25. Li C, Zhang X, Cheng L, Dai L, Xu F, Zhang J, Tian H, Chen X, Shi G, Li Y, Du T, Zhang $S$, Wei $Y$, Deng $H$ : RNA interference targeting human FAK and EGFR suppresses human non-small-cell lung cancer xenograft growth in nude mice. Cancer Gene Ther 2013, 20:101.

26. Camus S, Quevedo C, Menendez S, Paramonov I, Stouten PFW, Janssen RAJ, Rueb S, He S, Snaar-Jagalska BE, Laricchia-Robbio L, Belmonte JCI: Identification of phosphorylase kinase as a novel therapeutic target through high-throughput screening for anti-angiogenesis compounds in zebrafish. Oncogene 2012, 31:4333-4342.

27. Kawazu M, Ueno T, Kontani K, Ogita Y, Ando M, Fukumura K, Yamato A, Soda M, Takeuchi K, Miki Y, Yamaguchi H, Yasuda T, Naoe T, Yamashita Y, Katada T, Choi YL, Mano H: Transforming mutations of RAC guanosine triphosphatases in human cancers. Proc Natl Acad Sci U S A 2013, 110:3029-3034. 
28. Potiron VA, Roche J, Drabkin HA: Semaphorins and their receptors in lung cancer. Cancer Lett 2009, 273:1-14.

29. Feala JD, Cortes J, Duxbury PM, Piermarocchi C, McCulloch AD, Paternostro G: Systems approaches and algorithms for discovery of combinatorial therapies. Wires Syst Biol Med 2010, 2:181-193.

30. Pedregosa F, Varoquaux G, Gramfort A, Michel V, Thirion B, Grisel O, Blondel M, Prettenhofer P, Weiss R, Dubourg V: Scikit-learn Machine learning in Python. J Machine Learn Res 2011, 12:2825-2830.

doi:10.1186/1752-0509-8-74

Cite this article as: Tran et al:: Prediction of kinase inhibitor response using activity profiling, in vitro screening, and elastic net regression. BMC Systems Biology 2014 8:74.

\section{Submit your next manuscript to BioMed Central and take full advantage of:}

- Convenient online submission

- Thorough peer review

- No space constraints or color figure charges

- Immediate publication on acceptance

- Inclusion in PubMed, CAS, Scopus and Google Scholar

- Research which is freely available for redistribution 